\title{
Coherent Power Combination of Semiconductor Lasers Using Optical Phase-Lock Loops
}

\author{
Naresh Satyan, Wei Liang, Anthony Kewitsch, George Rakuljic, and Amnon Yariv, Life Fellow, IEEE
}

(Invited Paper)

\begin{abstract}
Heterodyne optical phase-lock loops (OPLLs) enable the precise electronic control over the frequency and phase of a semiconductor laser (SCL) locked to a "master" reference laser. One of the more interesting applications of OPLLs is the creation of coherent arrays by locking a number of "slave" SCLs to a common master laser. In this paper, we demonstrate the coherent power combination of various high-power semiconductor lasers using OPLLs in both the filled-aperture and tiled-aperture configurations. We further demonstrate the electronic control over the phase of each individual SCL using a voltage-controlled oscillator. It is feasible to combine a large number of SCLs using this approach, leading to compact, efficient, and cost-effective highpower and high-radiance optical sources.
\end{abstract}

Index Terms-Optical phase-locked loops (OPLLs), semiconductor laser (SCL) arrays, semiconductor lasers.

\section{INTRODUCTION}

$\mathbf{H}$ IGH-POWER lasers with ideal (diffraction limited) beam quality are sought after in a multitude of applications including scientific research, materials processing, and industrial applications, and research in this direction has been in progress ever since the invention of the laser. While high-power (few kilowatts single mode) fiber laser systems have been demonstrated, their output powers will ultimately be limited by nonlinear effects in the fiber and material damage. An alternate approach to obtain high-power laser radiation with excellent beam quality is by combining a large number of laser emitters with lower power outputs [1]-[3]. In particular, coherent beam combination (CBC) is a very promising approach to synthesize high-power optical sources with ideal beam quality. Various CBC schemes have been demonstrated by different groups, including evanescent wave coupling, self-organizing [2], injection locking [3], common resonator [4], and active feedback [5] approaches. While it is desirable to match the relative amplitudes, phases, polarizations, and pointing directions of all the component beams to achieve maximum efficiency in a $\mathrm{CBC}$

Manuscript received September 29, 2008; revised November 24, 2008. Current version published April 8, 2009. This work was supported by the Defense Advanced Research Projects Agency's (DARPA) Microsystems Technology Office (MTO).

N. Satyan is with the Department of Electrical Engineering, California Institute of Technology, Pasadena, CA 91125 USA (e-mail: naresh@ caltech.edu).

W. Liang is with OEwaves, Inc., Pasadena, CA 91106 USA (e-mail: wei.liang@oewaves.com).

A. Kewitsch and G. Rakuljic are with Telaris, Inc., Santa Monica, CA 90089 USA (e-mail: kewitsch@telarisinc.com; rakuljic@telarisinc.com).

A. Yariv is with the Department of Applied Physics and the Department of Electrical Engineering, California Institute of Technology, Pasadena, CA 91125 USA (e-mail: ayariv@ caltech.edu).

Digital Object Identifier 10.1109/JSTQE.2008.2011490

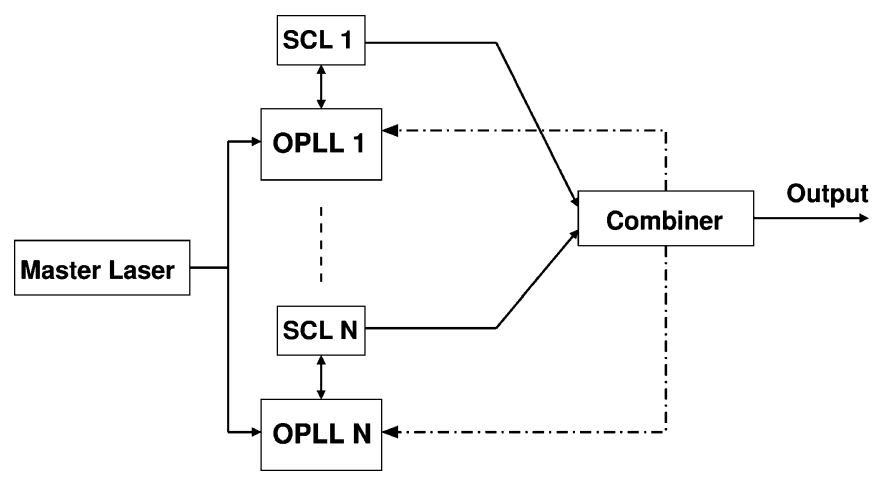

Fig. 1. Coherent power combining scheme using heterodyne SCL-OPLLs. Individual SCLs all lock to a common master laser, thus forming a coherent array. The outputs of the individual lasers are coherently combined to obtain a high-power single-mode optical beam.

scheme [1], the precise control over the optical phase offers the biggest challenge. Various active feedback approaches for phase control have been demonstrated, where the phase error between the combining beams is fed back to a servo system that includes phase actuators, which could be optical phase modulators [3], acousto-optic modulators [5], or fiber stretchers [6].

In this paper, we describe an alternative active feedback approach for CBC where an array of semiconductor lasers (SCLs) act as "slave" lasers that are phase-locked to a common "master" laser using heterodyne optical phase-lock loops (OPLLs). The outputs of the phase-locked slave SCLs are coherently combined to obtain a single high-power coherent optical beam, as shown in Fig. 1. The use of SCLs has many distinct advantages such as their compactness, high efficiency, low cost, and high output power, thereby making them attractive candidates for coherent power combination. The small size and high output powers of SCLs offer the potential for the combination of a number of SCLs on a single chip, leading to extremely compact high-power sources. Moreover, SCLs have a high-frequency modulation coefficient, which enables them to be used as current-controlled oscillators in OPLLs. The optical phase of each SCL in a coherent combination scheme can then be controlled electronically, which eliminates the need for optical phase or frequency shifters that are bulky, expensive, and require the use of large voltages.

This paper is organized as follows. We first review the concept of an SCL-OPLL and present our results of phase-locking various semiconductor lasers in Section II. In Section III, we present the results of the coherent power combination of different SCLs in the filled-aperture and tiled-aperture configurations. We demonstrate the all-electronic control of the optical phase 


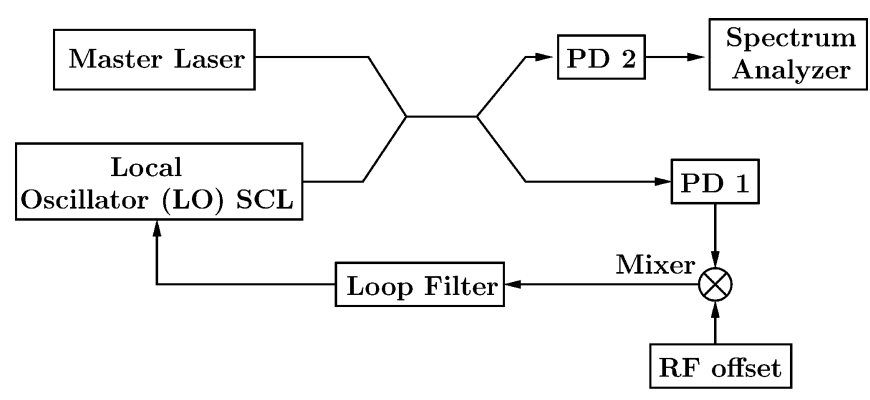

(a)

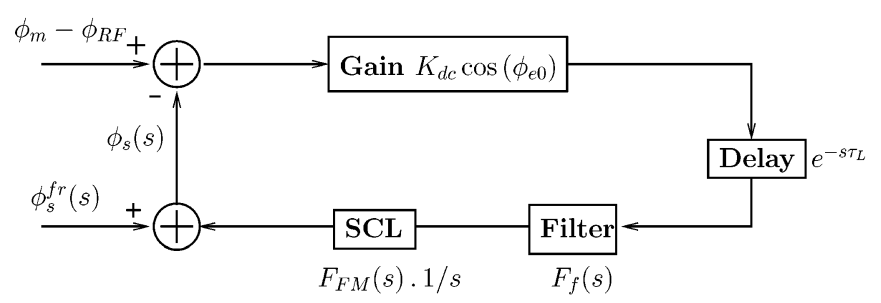

(b)

Fig. 2. (a) Schematic diagram of a heterodyne OPLL. (b) Model for phase noise propagation in an OPLL. PD: photodetector.

of a phase-locked SCL using a voltage -controlled oscillator (VCO), and show how this is used to correct for the variations in the differential optical paths traversed by the combining beams. We also discuss the scaling of the $\mathrm{CBC}$ scheme to combine the outputs of a large number of individual emitters and identify the important factors contributing to the overall power combination efficiency.

\section{OPTICAL PHASE-LOCK LOOPS}

\section{A. Theory}

The direct frequency modulation (FM) by current injection of an SCL makes it suitable as a current-controlled oscillator in an OPLL [7]-[9]. A schematic diagram of a heterodyne OPLL is shown in Fig. 2(a). The optical fields of the slave local oscillator (LO) SCL and the master laser are mixed in a photodetector PD1 and the resulting error signal is amplified, downconverted using an RF offset signal, filtered, and fed back to the SCL to complete the feedback loop. When the loop is in lock, the frequency of the SCL is related to the frequencies of the master laser and the RF offset signal by [10]

$$
\omega_{s}=\omega_{m}-\omega_{\mathrm{RF}} .
$$

The theoretical model for the propagation of the phase noise in the loop is shown in Fig. 2(b). The effect of the relative intensity noise (RIN) of the master and slave lasers on the phase of the locked laser is very small, and is therefore neglected in this analysis. $K_{\mathrm{dc}}$ is the dc open-loop gain, $F_{f}(s)$ and $F_{\mathrm{FM}}(s)$ are the transfer functions of the loop filter and the FM response of the SCL to input current, respectively, $s$ is the Laplace transform variable, with $s=j 2 \pi f$ on the imaginary axis of the $s$-plane, and $\phi_{e 0}$ is the steady-state phase error in the loop, and depends on the frequency difference $\Delta \omega$ between the master laser and the free running slave SCL, offset by the RF offset signal

$$
\phi_{e 0}=\sin ^{-1} \frac{\Delta \omega}{K_{\mathrm{dc}}} .
$$

The total small signal open-loop gain $G_{L}(s)$ given by

$$
G_{L}(s)=\frac{K_{\mathrm{dc}} \cos \phi_{e 0} F_{f}(s) F_{\mathrm{FM}}(s) e^{-s \tau_{L}}}{s} .
$$

Following a standard analysis [10] of the phase propagation in Fig. 2(b), we arrive at the following expression for the phase of the locked slave laser

$$
\begin{aligned}
\phi_{s}(s)= & \frac{G_{L}(s)}{1+G_{L}(s)}\left(\phi_{m}(s)-\phi_{\mathrm{RF}}(s)\right) \\
& +\frac{1}{1+G_{L}(s)} \phi_{s}^{\mathrm{fr}}(s)
\end{aligned}
$$

where $\phi_{m}(s), \phi_{\mathrm{RF}}(s)$, and $\phi_{s}^{\mathrm{fr}}(s)$ are the phase of the master laser, the RF offset signal, and the free running slave laser, respectively. From (4), we find that for frequencies smaller than the loop bandwidth, where $\left|G_{L}(s)\right| \gg 1$, the phase of the SCL tracks the phase of the master laser and the offset signal. For frequencies greater than the loop bandwidth, $\left|G_{L}(s)\right|<1$ and the SCL phase reverts to its free running value. The measure of the efficiency of the phase-locking is given by the variance of the residual phase noise of the OPLL, viz., the phase difference between the slave and the master laser, offset by the phase of the RF signal

$$
\sigma_{\phi}^{2}=\left\langle\left(\phi_{m}-\phi_{\mathrm{RF}}-\phi_{e 0}-\phi_{s}\right)^{2}\right\rangle .
$$

Here, $\phi_{e 0}$ is the steady-state dc phase error signal in the loop, and is given by (2).

Consider a simple first-order OPLL with no delay and closedloop bandwidth $B$, i.e., $G_{L}(s)=B / s$. Let the master laser and the free running slave laser have Lorenzian lineshapes with fullwidth at half-maximums (FWHMs) $\Delta \nu_{m}$ and $\Delta \nu_{s}$, respectively. Using (4) and (5), and neglecting the phase noise of the offset $\mathrm{RF}$ signal, the variance of the residual phase noise is given by

$$
\sigma_{\phi}^{2}=\frac{\pi\left(\Delta \nu_{m}+\Delta \nu_{s}\right)}{B} .
$$

From (6), it is important to have a loop bandwidth much larger than the laser linewidths so that the residual phase noise remains small. The residual phase noise degrades loop performance and contributes to a reduced power combination efficiency, as shown in Section III. Further, it is desirable that the holding range of the loop, defined as the range over which the free running frequency difference $\Delta \omega$ can vary before the loop loses lock, be as large as possible. The holding range of the loop is determined by the dc gain $K_{\mathrm{dc}}$ in (3), which, in turn, is ultimately limited by the loop bandwidth. The loop bandwidth of SCL-OPLLs is limited by two factors, viz., the loop propagation delay $\tau_{L}$ [11], [12] and the thermally induced phase reversal of the FM response of single-section SCLs [13]. These bandwidth constraints can be relaxed by the use of active and passive loop filters [10], and by the use of microoptics and multiple-section distributed feedback (DFB) lasers with a superior FM response [9]. 
TABLE I

OPLL RESULTS FOR VARIOUS SCLS

\begin{tabular}{|c|c|c|c|c|c|}
\hline $\begin{array}{c}\text { Slave } \\
\text { SCL }\end{array}$ & $\begin{array}{c}\lambda \\
(\mathrm{nm})\end{array}$ & $\begin{array}{c}\text { SCL } \\
\text { power }\end{array}$ & $\begin{array}{c}\text { SCL -20 dB } \\
\text { linewidth }\end{array}$ & $\begin{array}{c}\text { Master } \\
\text { Laser }\end{array}$ & $\begin{array}{c}\sigma_{\phi} \\
(\mathrm{rad})\end{array}$ \\
\hline DFB & 1539 & $60 \mathrm{~mW}$ & $4.4 \mathrm{MHz}$ & Fiber Laser & 0.32 \\
\hline MOPA & 1548 & $1 \mathrm{~W}$ & $4.8 \mathrm{MHz}$ & Tunable Laser & 0.28 \\
\hline ECL & 1064 & $100 \mathrm{~mW}$ & $2 \mathrm{MHz}$ & Fiber Laser & 0.12 \\
\hline
\end{tabular}

\section{B. Experiment}

The OPLL experiment depicted in Fig. 2(a) was performed using a DFB laser (JDSU) [14], a high-power master oscillator power amplifier (MOPA) laser (QPC Lasers, Inc.) [15] and an external cavity laser (ECL, Innovative Photonic Solutions) [16], with parameters summarized in Table I. The MOPA laser has a DFB oscillator section followed by a tapered amplifier section, and its frequency/phase is tuned by varying the current into the oscillator section. Closed-loop control using thermoelectric coolers is used to stabilize the temperature of the SCLs. The injection current and temperature of the SCLs are tuned to set their free running frequency to be nominally equal to that of the master laser offset by the RF. A narrow-linewidth fiber laser (NP Photonics) or a tunable laser (Agilent) was used as the master laser in the experiments. The power of the master laser coupled into the loop can be very small $(\sim-10 \mathrm{dBm})$ since this can be electronically compensated by increasing the loop gain. A large number of SCLs can therefore be locked to the same master laser, enabling the creation of multiple coherent sources. The outputs of the fiber-coupled slave SCL and the master laser are combined using a fiber coupler, and mixed on a high-speed photodetector [New Focus 1544B, PD1 in Fig. 2(a)]. The output of the photodetector is amplified and mixed with an RF offset signal in the range $0.8-2 \mathrm{GHz}$. The downconverted error signal at the mixer output is filtered using a combination of active and passive filters to improve the dc gain and the loop bandwidth, and is fed back into the slave SCL to complete the loop.

The spectrum of the beat signal between the phase-locked SCL and the master laser is measured on an RF spectrum analyzer. The experimentally measured beat spectra for the OPLL with the high-power MOPA and the external cavity slave SCLs are shown in Fig. 3. The rms residual phase error in the OPLL is calculated from the beat spectrum using the relation

$$
\sigma_{\phi}=\sqrt{\frac{P_{n}}{P_{s}}}
$$

where $P_{n}$ is the noise power obtained by integrating the measured spectral density over the entire frequency range except the carrier frequency and $P_{s}$ is the power of the carrier signal. The experimentally measured rms phase errors for the various OPLLs are tabulated in Table I.

The typical loop propagation delay in our fiber-based OPLL experiments is about $5 \mathrm{~ns}$, which corresponds to a delay-limited loop bandwidth of about $50 \mathrm{MHz}$. The limitation imposed on the loop bandwidth by the loop propagation delay can be relaxed by using a free-space OPLL with miniature optics, and high-speed ICs for the electronic components in order to reduce rise time and propagation delay. However, the bandwidth

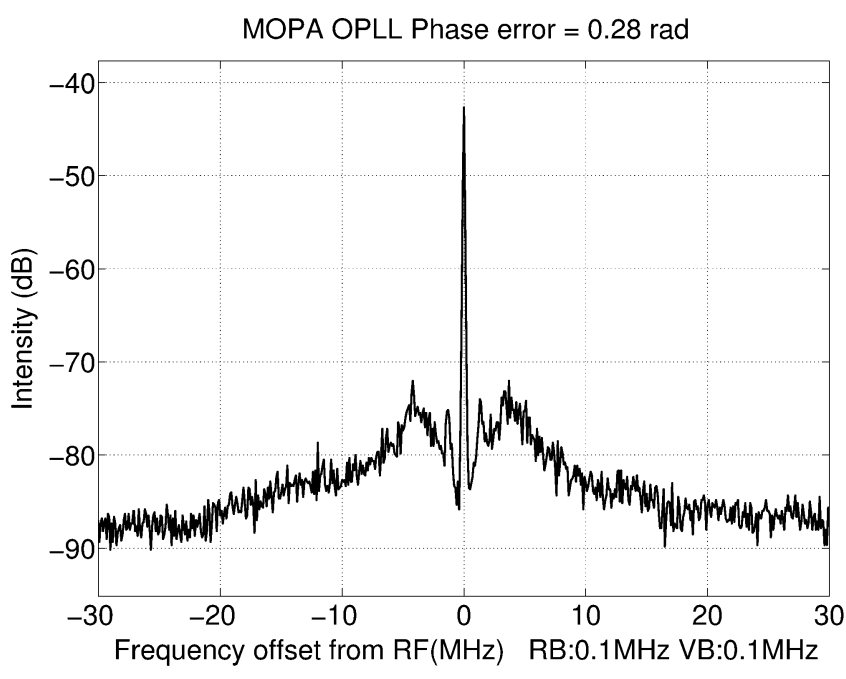

(a)

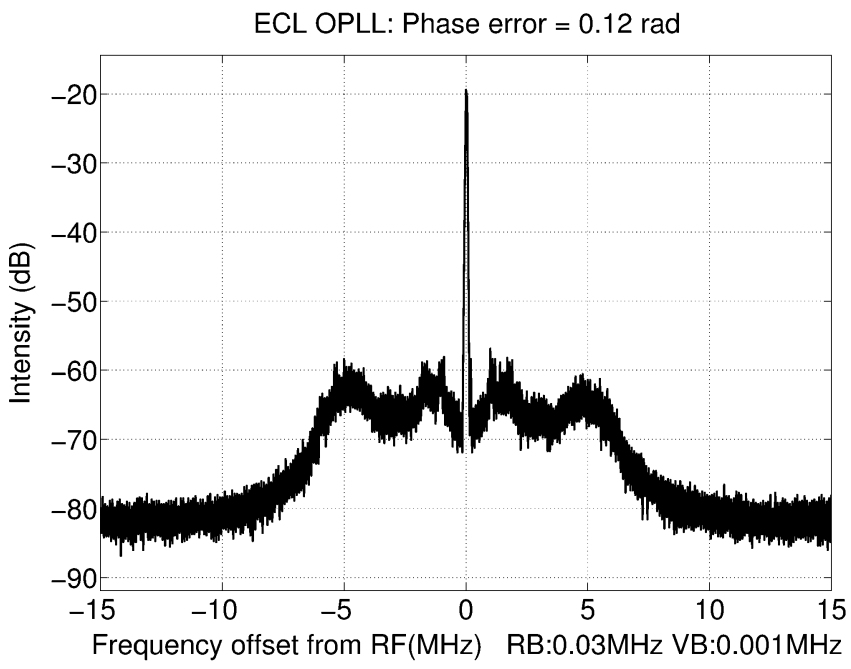

(b)

Fig. 3. Measured spectrum of the beat signal between the master laser and the phase-locked slave SCL. (a) High-power MOPA laser at $1548 \mathrm{~nm}$. (b) ECL at $1064 \mathrm{~nm}$.

of the phase-locking in our OPLL experiments was limited to 3-7 MHz due to the phase crossover of the FM response [13] of the SCLs used in the experiments. The bandwidth was increased to $\sim 10 \mathrm{MHz}$ and the holding range was increased to more than $\pm 3 \mathrm{GHz}$ by the use of passive and active loop filters. An aided acquisition circuit, designed by our collaborators at the University of Southern California (USC) [17], was implemented to increase the acquisition range from $\sim \pm 10 \mathrm{MHz}$ to $\sim \pm 1 \mathrm{GHz}$.

\section{Coherent Power Combination Using OPLLs}

As described in Section I, when an array of SCLs are all phase-locked to the same master laser in heterodyne OPLLs with the same RF offset, they all acquire the same frequency and phase as long as the loop bandwidths are sufficiently high. The outputs of these lasers can therefore be coherently combined to produce a single high-power optical beam. Coherent power combination results in optical beams with superior beam quality and larger peak intensities as compared to incoherent power 


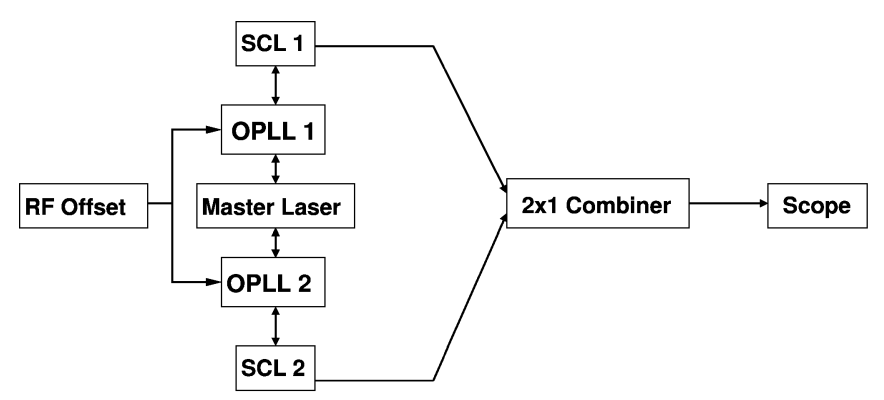

(a)

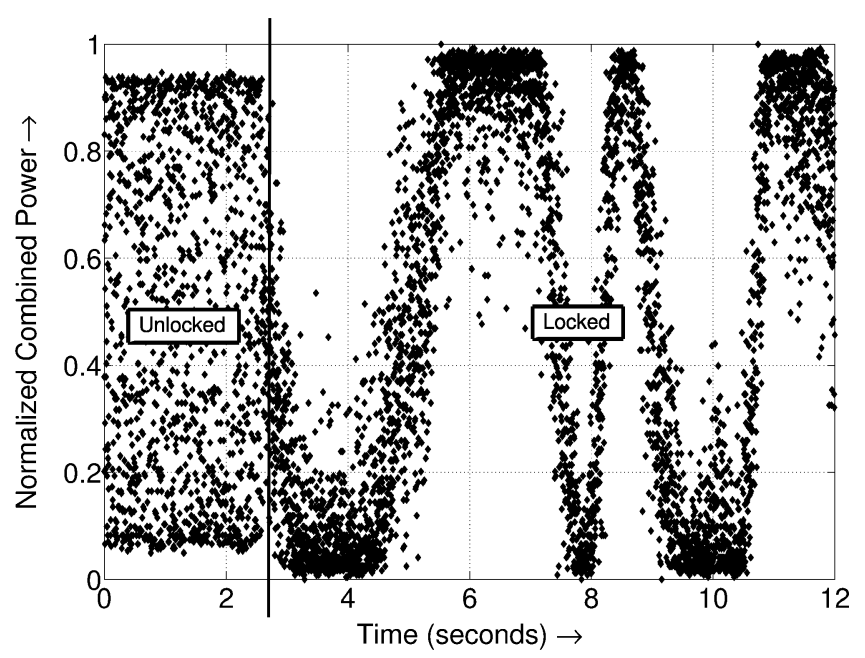

(b)

Fig. 4. (a) Coherent combination schematic. Two SCLs are locked to a common master laser at a common offset and the combined output is measured on an oscilloscope. (b) Experimentally measured combined power using two high-power MOPAs as slave lasers phase-locked to a common master laser. For $t<2.5 \mathrm{~s}$, one of the OPLLs is not in lock, and the result is the incoherent power addition of the two lasers.

addition. There are two approaches to CBC [1]: 1) the filledaperture approach where multiple beams are combined into a single beam using a beam combiner and 2) the tiled-aperture approach, where the outputs of the individual emitters are adjacent to each other. One of the key aspects in either approach is the control over the relative phases of the individual emitters at the beam combiner. In this section, we present our results of the coherent combination of two SCLs using both the aforementioned approaches. When the outputs of two lasers with powers $P_{0}$ are coherently combined, the total power obtained is

$$
P_{c}=P_{0}(1+\cos \theta)
$$

where $\theta$ is the phase difference between the two combining beams. For maximal power combining efficiency, we need $\theta=0$. Variations in the optical paths traversed by the different combining beams result in a deviation from this ideal value, leading to a reduced combining efficiency. It is therefore necessary to correct for these variations.

\section{A. Filled-Aperture Combination}

A schematic of the filled aperture power combination experiment is shown in Fig. 4(a). Two slave SCLs are locked to a common master laser using heterodyne OPLLs, as shown in Fig. 2(a). A common RF offset signal is fed to each loop. It is only necessary to use a small fraction of the SCL output in the feedback loop, and the remaining power is used for power combination. The outputs of the two SCLs are combined using a $2 \times 1$ fiber combiner, and the output is measured on an oscilloscope. The result of the experiment with the high-power MOPAs is shown in Fig. 4(b). For time $<2.5 \mathrm{~s}$, one of the lasers is unlocked, and the resultant incoherent addition results in high-frequency oscillations on the oscilloscope at the (time-varying) beat frequency between the two SCLs. When both the loops are in lock, the result is a "dc" signal that varies very slowly (on the time scale of a few seconds). This slow drift is due to the change in the differential path lengths traversed by the combining beams. In addition to the slow drift, the combined power signal also shows fast variations due to the residual phase noise between the two combined beams. The rms value of the residual phase error is estimated from the fast variations in the measurement in Fig. 4(b) to be about 0.39 rad. This corresponds to a residual phase error of $0.39 / \sqrt{2}=0.28 \mathrm{rad}$ in each OPLL, which is in very good agreement with the measured value in Table I. We present shortly a novel electronic feedback scheme developed to correct for the slow drift in the relative phase between the optical beams.

\section{B. Phase Control Using a VCO}

The variations in the differential optical paths traversed by combining beams is traditionally controlled using a piezoelectric fiber stretcher, an acousto-optic modulator, or an optical phase modulator [3], [5], [6]. The phase of the phase-locked SCL in a heterodyne OPLL follows the phase of the RF offset signal, and this allows for the electronic control over the optical phase. The phase of the RF offset signal can be tuned using an RF phase shifter, but this method has the same shortcomings as an optical phase shifter, i.e., insufficient dynamic range to correct for large phase errors. Typical optical or RF phase modulators have a dynamic range of $2 \pi \mathrm{rad}$, and complicated reset circuitry is often necessary to increase the dynamic range. Here, we present an alternative phase-control scheme where the correction signal is provided by an electronic VCO. In addition to acting as an integrating phase shifter with practically infinite dynamic range, the VCO also provides the RF offset signal to the heterodyne OPLL.

A schematic of the power combining experiment with the VCO correction loop is shown in Fig. 5(a). Two SCLs are phaselocked to a common master laser using heterodyne OPLLs. While an RF source provides a fixed offset signal to one OPLL, the offset signal to the other OPLL is provided by a VCO. The nominal free-running frequency of the VCO is chosen to be equal to the frequency of the RF source. The outputs of the two lasers are combined using a $2 \times 2$ fiber coupler. One of the outputs of the coupler (the "combined" output) is observed on an oscilloscope, while the other output (the "null" output) is amplified and fed into the control port of the VCO. The measured combined power signal, with and without the VCO control loop, in the power combining experiment using ECLs is shown in Fig. 5(b). A stable power combining efficiency of $94 \%$ 


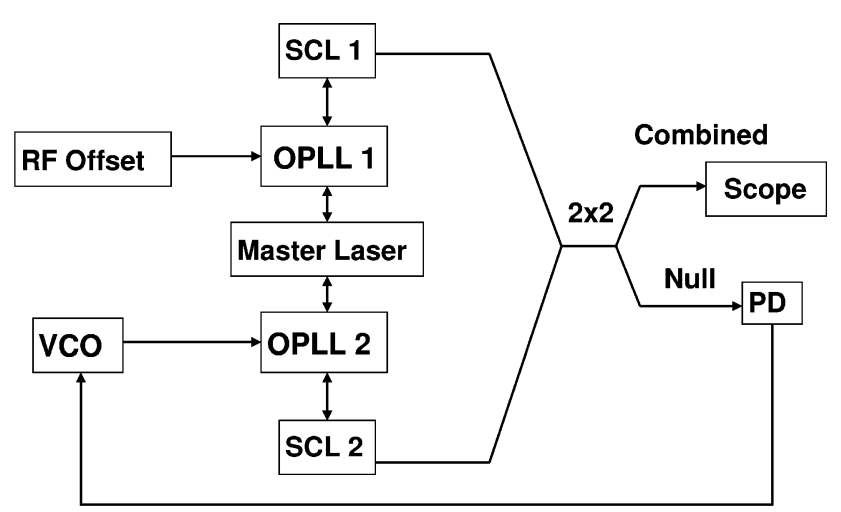

(a)

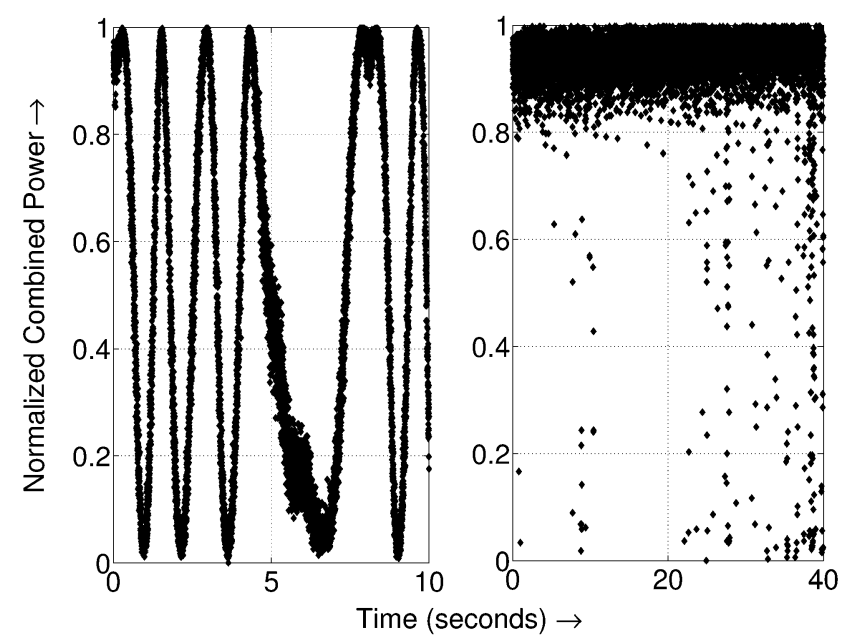

(b)

Fig. 5. (a) Schematic of the coherent combination experiment with additional electronic phase control. A VCO provides the offset signal to the second OPLL, and also acts as an integrating phase shifter to correct for variations in the differential optical path length. (b) Experimentally measured combined power using external cavity SCLs at $1064 \mathrm{~nm}$, (left) without and (right) with the VCO loop connected. The power combining efficiency with the VCO loop is $94 \%$.

is obtained using the VCO phase correction loop. This efficiency is mainly limited by the jitter of the free running frequency of the VCO used in the experiment and not by the residual phase noise in the OPLL, and can therefore be further improved by the use of cleaner VCOs. The VCO frequency jitter is also responsible for the occasional cycle slips seen in Fig. 5(b).

A steady-state analysis of the VCO feedback scheme is presented in [18] and is briefly summarized here. The null output of the power combiner, given by $P_{0}(1-\cos \theta)$, is fed to the $\mathrm{VCO}$, which acts as an integrating phase shifter. If the total dc gain in this branch of the feedback system is $K_{v}$, the steady-state value of the phase difference $\theta$ is given by

$$
\theta_{0}=\cos ^{-1}\left(1-\frac{\omega_{\mathrm{RF}}-\omega_{v}}{K_{v}}\right)
$$

where $\omega_{v}$ is the free running frequency of the VCO. From (8) and (9), it is clear that a large $K_{v}$ is desirable so that $\theta_{0}$ is small, and a higher efficiency is achieved. We next linearize the phase difference $\theta$ about the steady-state value $\theta_{0}$. The small-signal model for the VCO control system is shown in Fig. 6. SCL1

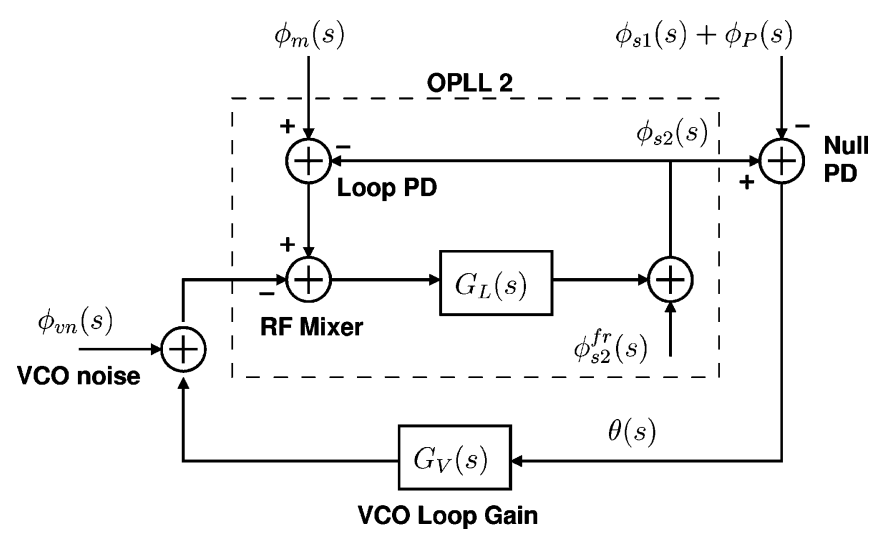

Fig. 6. Small-signal phase model for the power combining scheme with the additional VCO loop. SCL1 is locked to the master laser in OPLL1, and is not shown here. PD: photodetector.

is locked to the master laser in OPLL1 and its phase noise $\phi_{s 1}$ is given by (4). The free running phase noise of the VCO and the slave SCL2 are denoted by $\phi_{v n}$ and $\phi_{s 2}^{\mathrm{fr}}$, respectively. The variation in the differential path lengths traversed by the outputs of SCL1 and SCL2 produces a phase noise at the fiber combiner, and this noise has the Laplace transform $\phi_{P}$. The OPLL openloop gain is the same as (3), and the gain $G_{V}(s)$ in the VCO branch is

$$
G_{V}(s)=\frac{K_{v} \sin \theta_{0} e^{-s \tau_{v}}}{s}
$$

where $\tau_{v}$ is the delay in the VCO branch (from the null photodetector to the RF mixer) and $\theta_{0}$ is as in (9). Note that there is a tradeoff in the choice of the value of $\theta_{0}$ : a smaller $\theta_{0}$ results in a higher power combination efficiency, but also results in a lower loop gain. The reduction in loop gain can be compensated by increasing the dc gain $K_{v}$.

The model in Fig. 6 can be solved for the variation in the output phase $\theta(s)$ to yield

$$
\theta(s)=\frac{1}{1+G_{L}+G_{L} G_{V}}\left(\begin{array}{c}
\phi_{s 2}^{\mathrm{fr}}+G_{L}\left(\phi_{m}-\phi_{v n}\right) \\
-\left(1+G_{L}\right)\left(\phi_{s 1}+\phi_{P}\right)
\end{array}\right) .
$$

The argument $s$ has been dropped from all the terms on the right-hand side. We substitute for $\phi_{s 1}(s)$ using (4) to obtain

$$
\theta(s)=\frac{1}{1+G_{L}+G_{L} G_{V}}\left(\begin{array}{c}
\left(\phi_{s 2}^{\mathrm{fr}}-\phi_{s 1}^{\mathrm{fr}}\right) \\
+G_{L}\left(\phi_{\mathrm{RF}}-\phi_{v n}\right) \\
-\left(1+G_{L}\right) \phi_{P}
\end{array}\right) .
$$

To obtain some physical insight into the previous equation, we note that the delay in the VCO loop $\tau_{v}$ is typically much larger than the OPLL delay. This limits the VCO open-loop gain $G_{V}$ so that the approximation $\left|G_{L}\right| \gg\left|G_{V}\right|$ holds at all frequencies. The denominator in (12) can then be expressed as $\left(1+G_{L}\right)(1+$ $G_{V}$ ), and the system can be regarded as the combination of three phase-lock loops [16] as follows. The slave lasers SCL1 and SCL2 are locked to the master laser using heterodyne OPLLs at offsets given by $\omega_{\mathrm{RF}}$ and $\omega_{v}$, respectively; the open-loop 


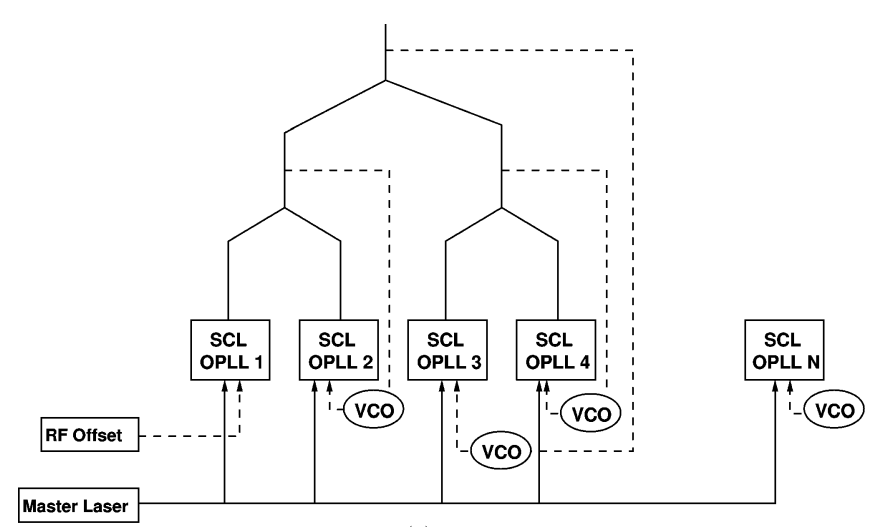

(a)

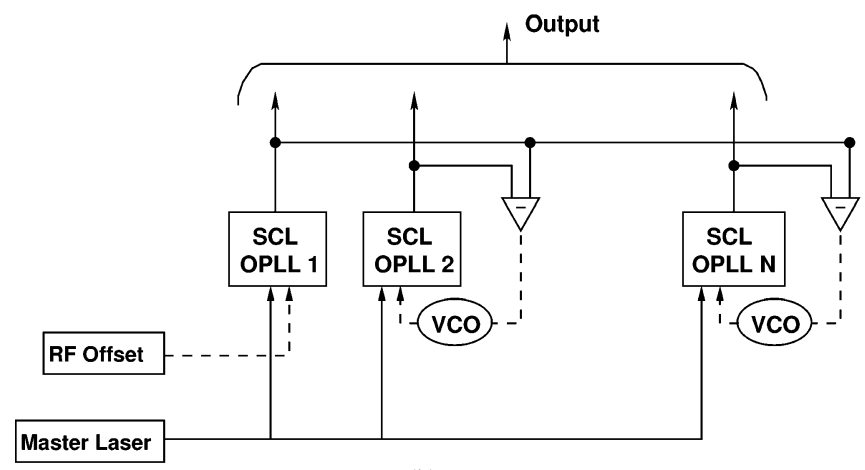

(b)

Fig. 7. Binary tree configuration for the power combination of a number of SCLs locked to a common master laser in the (a) filled-aperture and (b) tiledaperture configurations.

gains of these OPLLs are given by $G_{L}(s)$. The VCO frequency $\omega_{v}$ is then locked to the RF offset frequency $\omega_{\mathrm{RF}}$ in a third "outer" PLL with open-loop gain $G_{V}(s)$. The laser phase noise is suppressed by the OPLLs, while the phase jitter of the VCO and the variation $\phi_{P}$ in the differential optical path length are suppressed by the third PLL.

\section{Combining Efficiency}

The power combination approach presented before can be scaled to a large number of lasers using a binary tree configuration, as shown in Fig. 7(a). Fiber amplifiers can be used at the output of each slave SCL to increase the overall combined power. The addition of fiber amplifiers increases the delay in the outer VCO loop, but the resultant bandwidth is still sufficient to correct for the slow fluctuations in the differential optical path length introduced by the amplifiers. The additional phase noise introduced into the system due to amplified spontaneous emission (ASE) in fiber amplifiers is negligible when high-power narrow-linewidth lasers are used [19]-[21]. We have demonstrated the coherent combination of four fiber-amplified (35-40 W) SCLs using this approach to achieve a coherent and diffraction-limited power output of $\sim 110 \mathrm{~W}$ [22].

The overall power combining efficiency is affected by the intensity noise, relative polarizations, and relative phase error between the combining beams, but is mainly limited by the phase noise of the combining beams. From (8), assuming that the deviations of the relative phase about the ideal value of zero are small, the efficiency of combining two optical beams is given by

$$
\eta=\frac{P_{c}}{2 P_{0}} \approx 1-\frac{\left\langle\theta^{2}\right\rangle}{4} .
$$

The mean-squared value of the relative phase $\left\langle\theta^{2}\right\rangle$ has two important contributions: 1) the steady-state phase $\theta_{0}$ given by (9) and 2) the residual phase noise of both the SCLs and the VCO given by (12). The value of $\theta_{0}$ can be reduced by the use of cleaner VCOs and also loop filters to increase the dc gain $K_{v}$. The residual phase noise of the SCLs can be reduced by increasing the OPLL loop bandwidth. When the scheme is extended to combine a large number $N$ of SCLs as in Fig. 7(a), the important contributions to deviation from ideality of the overall combining efficiency are: 1) the residual OPLL phase noise; 2) the frequency jitter of the VCOs; and 3) the phase-front deformations caused by the optical elements used for beam combining. The effect of each of these factors is analyzed in detail in [16].

\section{Tiled-Aperture Combination}

The OPLL approach also lends itself to the tiled-aperture combination of SCLs [23]. A schematic diagram of the tiledaperture coherent power combining experiment using discrete DFB SCLs is shown in Fig. 8(a). The optical signals of the two DFB SCLs are collimated and placed next to each other, and the far-field pattern is observed using an IR camera placed at the focal plane of a convex lens. A small fraction of each slave beam is tapped by a beam splitter and is used to lock the SCLs to a common master laser using heterodyne OPLLs. An RF phase shifter is used to adjust the phase of one of the RF offset signals, and hence, the differential phase between the two slave beams.

The measured far-field patterns of the incoherently and the coherently added beams are shown in Fig. 8(b). The horizontal intensity distributions of the far-field patterns are shown in Fig. 8(c). Incoherent addition refers to the case where one of the OPLLs is not in lock. When both the OPLLs are in lock, the two slave beams are coherently added and interference fringes are seen in the far-field pattern. Comparing the beam resulting from in-phase coherent addition (circles) to the incoherently added beam (triangles), we see that the width of the central lobe is narrowed almost by a factor of 2 and the peak intensity increases by a factor of $\sim 75 \%$, leading to a Strehl ratio of $\sim 0.87$. The deviation of the Strehl ratio from the ideal value of unity is due to the residual phase noise in the OPLLs and the mismatch between the polarizations of the slave beams. Further, the principle of beam steering is demonstrated by varying the phase of one of the RF offset signals from 0 to $\pi$. The optical phase of the slave SCL exactly follows the phase of the RF offset signal, and the phase difference between the combining beams thus varies from 0 to $\pi$ rad, resulting in a shift in the interference fringes, as seen in Fig. 8(b) and (c). Note that the phase-locking in Fig. 8(a) is performed by comparing the phases of master laser and the two slave SCLs at the output, and hence, the variation in the relative optical path traversed by the combining beams is corrected by the two OPLLs. However, this scheme may be impractical in certain configurations, e.g., if fiber amplifiers are 


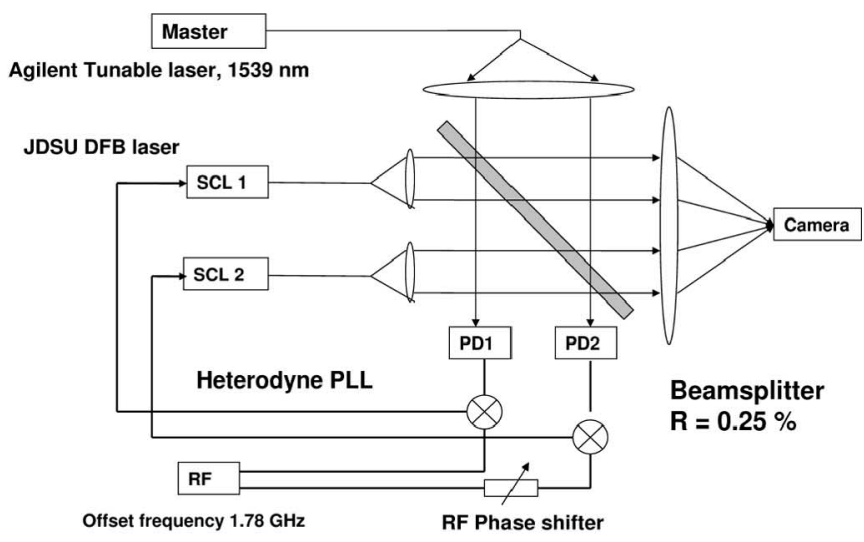

(a)

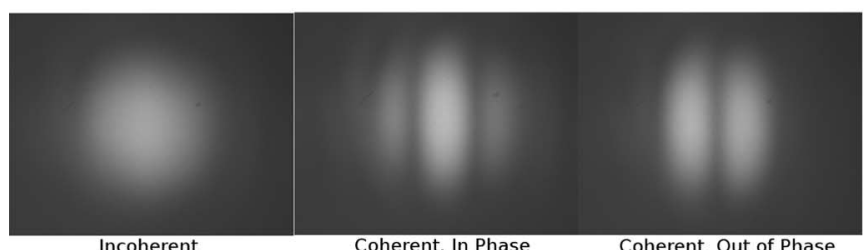

(b)

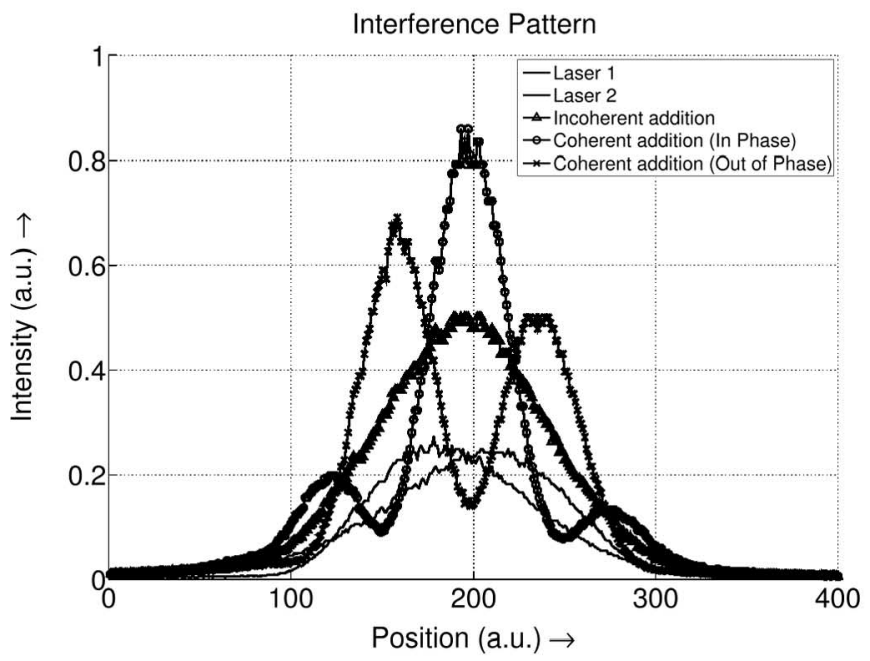

(c)

Fig. 8. (a) Schematic of the tiled-aperture coherent power combination experiment. (b) Far-field profiles of the incoherently and coherently added beams as measured on the IR camera. (c) Horizontal intensity distributions of the measured far-field profiles.

used at the outputs of the slave SCLs, they introduce additional delays and drastically reduce the loop bandwidth. In such cases, an additional VCO loop, analogous to the filled aperture case, can be used to correct the phase noise due to variations in the differential optical path, as shown in Fig. 7(b). An RF oscillator is used to provide the RF offset signal to OPLL 1, while VCOs are used as the offset signals in OPLLs 2 through $N$. The control voltage to the VCO in OPLL $j$ is given by the beat signal between the outputs of SCL 1 and SCL $j$, which can be detected using a low-speed photodetector. The results of a tiledaperture combination experiment with two DFB SCLs with an additional VCO loop have been reported in [23]. This approach to the tiled-aperture coherent combination of arrays of SCLs using OPLLs can lead to the development of phase-controlled apertures, with applications in electronic beam steering, beam shaping, adaptive wavefront correction, etc.

\section{CONCLUSION}

We have presented an all-electronic active feedback approach for the coherent power combination of SCLs using OPLLs. An array of SCLs locked to a common master laser have the same frequency and phase, and can be coherently combined. The phase of the combining SCLs is further controlled using a $\mathrm{VCO}$, to compensate for differential path length variations of the combining beams. We have demonstrated the coherent combination of various high-power SCLs using this approach, and have achieved a stable power combining efficiency of $94 \%$. The electronic feedback scheme demonstrated eliminates the need for optical phase or frequency shifters. The OPLL approach can be used to combine SCLs in both the filled-aperture and tiledaperture configurations. It is possible to obtain coherent and diffraction-limited power of tens of kilowatts by the use of fiber amplifiers to amplify the outputs of an array of phase-locked SCLs. When scaled to a large number of SCLs, the overall power combination efficiency is likely to be limited by the less-thanunity fill factor for the tiled-aperture configuration, and by VCO jitter and phase-front deformations in the filled-aperture case.

The coherent power combining of tens of high-power SCLs on a single chip in the tiled-aperture configuration has the potential to enable compact and inexpensive high-power sources. The practical realization of these sources will depend on a number of factors. The loop bandwidth of an OPLL with a single-section SCL is limited by the nonuniform thermal frequency response of the laser to $<10 \mathrm{MHz}$, which, in turn, places a restriction on the linewidth of the SCL, as given by (6). Therefore, narrow-linewidth SCLs such as external cavity or DFB lasers, or multiple-section lasers with a larger FM bandwidth will need to be used as the array elements. The fabrication of the various electronic components of an OPLL such as the mixer, amplifiers, filters, etc., on a single IC has been demonstrated [17], and this needs to be extended to multiple OPLLs. Integrated waveguides and photodetectors for broadband receivers based on OPLLs have also been demonstrated [24]; the fabrication of an array of such detectors is necessary to enable a coherent OPLL array. While it is difficult to phase-lock standard packaged diode laser bars using this scheme, we believe that it is feasible to use integrated optical waveguides to combine the outputs of many discrete phaselocked SCLs residing on a single chip, to form a single coherent aperture with narrow spacing between adjacent emitters and electronic control over the phase of each emitter in the aperture.

\section{ACKNOWLEDGMENT}

This paper was a collaborative effort between Caltech, Telaris, Inc., and the University of Southern California, and was supported by the Defense Advanced Research Projects Agency (DARPA) (Microsystems Technology Office (MTO), Architecture for Diode High Energy Laser Systems (ADHELS) Program). The authors gratefully acknowledge their collaborators F. Aflatouni and Prof. Hashemi at the USC. 


\section{REFERENCES}

[1] T. Y. Fan, "Laser beam combining for high-power, high-radiance sources," IEEE J. Sel. Topics Quantum Electron., vol. 11, no. 3, pp. 567-577, May/Jun. 2005.

[2] A. Shirakawa, T. Saitou, T. Sekiguchi, and K. Ueda, "Coherent addition of fiber lasers by use of a fiber coupler," Opt. Exp., vol. 10, no. 21, pp. 1167-1172, 2002.

[3] L. Bartelt-Berger, U. Brauch, A. Giesen, H. Huegel, and H. Opower, "Power-scalable system of phase-locked single-mode diode lasers," Appl. Opt., vol. 38, no. 27, pp. 5752-5760, 1999.

[4] Y. Kono, M. Takeoka, K. Uto, A. Uchida, and F. Kannari, "A coherent all-solid-state laser array using the talbot effect in a three-mirror cavity," IEEE J. Quantum Electron., vol. 36, no. 5, pp. 607-614, May 2000.

[5] S. J. Augst, T. Y. Fan, and A. Sanchez, "Coherent beam combining and phase noise measurements of ytterbium fiber amplifiers," Opt. Lett., vol. 29, no. 5, pp. 474-476, 2004.

[6] C. X. Yu, J. E. Kansky, S. E. J. Shaw, D. V. Murphy, and C. Higgs, "Coherent beam combining of large number of PM fibres in 2-D fibre array," Electron. Lett., vol. 42, no. 18, pp. 1024-1025, 2006.

[7] R. C. Steele, "Optical phase-locked loop using semiconductor laser diodes," Electron. Lett., vol. 19, no. 2, pp. 69-71, 1983.

[8] A. Yariv, "Dynamic analysis of the semiconductor laser as a currentcontrolled oscillator in the optical phased-lock loop: Applications," Opt. Lett., vol. 30, no. 17, pp. 2191-2193, 2005.

[9] L. N. Langley, M. D. Elkin, C. Edge, M. J. Wale, U. Gliese, X. Huang, and A. J. Seeds, "Packaged semiconductor laser optical phase-locked loop (OPLL) for photonic generation, processing and transmission of microwave signals," IEEE Trans. Microw. Theory Tech., vol. 47, no. 7, pp. 1257-1264, Jul. 1999.

[10] F. M. Gardner, Phaselock Techniques, 3rd ed. New York: Wiley, 2005.

[11] M. Grant, W. Michie, and M. Fletcher, "The performance of optical phaselocked loops in the presence of nonnegligible loop propagation delay," $J$. Lightw. Technol., vol. 5, no. 4, pp. 592-597, Apr. 1987.

[12] R. T. Ramos and A. J. Seeds, "Delay, linewidth and bandwidth limitations in optical phase-locked loop design," Electron. Lett., vol. 26, no. 6, pp. 389-391, Mar. 1990.

[13] P. Corrc, O. Girad, and I. F. de Faria, Jr., "On the thermal contribution to the FM response of DFB lasers: Theory and experiment," IEEE J. Quantum Electron., vol. 30, no. 11, pp. 2485-2490, Nov. 1994.

[14] W. Liang, A. Yariv, A. Kewitsch, and G. Rakuljic, "Coherent combining of the output of two semiconductor lasers using optical phase-lock loops," Opt. Lett., vol. 32, no. 4, pp. 370-372, 2007.

[15] W. Liang, N. Satyan, A. Yariv, A. Kewitsch, G. Rakuljic, F. Aflatouni, H. Hashemi, and J. Ungar, "Coherent power combination of two masteroscillator-power-amplifier (MOPA) semiconductor lasers using optical phase lock loops," Opt. Exp., vol. 15, no. 6, pp. 3201-3205, 2007.

[16] W. Liang, N. Satyan, F. Aflatouni, A. Yariv, A. Kewitsch, G. Rakuljic, and H. Hashemi, "Coherent beam combining with multilevel optical phaselocked loops," J. Opt. Soc. Amer. B, Opt. Phys., vol. 24, no. 12, pp. 2930 2939, 2007.

[17] F. Aflatouni, O. Momeni, and H. Hashemi, "A heterodyne phase locked loop with $\mathrm{GHz}$ acquisition range for coherent locking of semiconductor lasers in $0.13 \mu \mathrm{m}$ CMOS," in Proc. IEEE Custom Integr. Circuits Conf. (CICC 2007), Sep., pp. 463-466.

[18] N. Satyan, W. Liang, F. Aflatouni, A. Yariv, A. Kewitsch, G. Rakuljic, and H. Hashemi, "Phase-controlled apertures using heterodyne optical phaselocked loops," IEEE Photon. Technol. Lett., vol. 20, no. 11, pp. 897-899, Jun. 2008.

[19] E. Desurvire, Erbium-Doped Fiber Amplifiers: Principles and Applications. New York: Wiley, 1994.

[20] L. Moller, "Novel aspects of spectral broadening due to fiber amplifier phase noise," IEEE J. Quantum Electron., vol. 34, no. 9, pp. 1554-1558, Sep. 1998.

[21] E. Rochat and R. Dandliker, "New investigations on the effect of fiber amplifier phase noise," IEEE J. Sel. Topics Quantum Electron., vol. 7, no. 1, pp. 49-54, Jan./Feb. 2001.

[22] A. Kewitsch, G. Rakuljic, N. Satyan, W. Liang, and A. Yariv, "Coherent power combination of fiber amplified phase-locked semiconductor lasers for output power $>100 \mathrm{~W}$," to be published.

[23] W. Liang, N. Satyan, A. Yariv, A. Kewitsch, and G. Rakuljic, "Tiledaperture coherent beam combining using optical phase-lock loops," Electron. Lett., vol. 44, no. 14, pp. 875-876, Jul. 2008.

[24] H.-F. Chou, A. Ramaswamy, D. Zibar, L. A. Johansson, J. E. Bowers, M. Rodwell, and L. A. Coldren, "Highly linear coherent receiver with feedback," IEEE Photon. Technol. Lett., vol. 19, no. 12, pp. 940-942, Jun. 2007.
Naresh Satyan received the B.Tech. degree in electrical engineering from the Indian Institute of Technology Madras, Chennai, India, in 2005, and the M.S degree in electrical engineering in 2007 from California Institute of Technology (Caltech), Pasadena, where he is currently working toward the Ph.D. degree in electrical engineering.

His current research interests include optical phase-lock loops, semiconductor lasers, coherent optics, RF photonics, and optoelectronics.

Wei Liang received the B.S. degree from Tsinghua University, Beijing, China, in 2001, and the M.S. and Ph.D. degrees in applied physics from California Institute of Technology (Caltech), Pasadena, in 2003 and 2008, respectively.

$\mathrm{He}$ is currently with OEwaves, Inc., Pasadena, CA. His current research interests include phase- and frequency-locking of lasers, RF photonics, and coherent optics.

Anthony Kewitsch received the B.S. degree in electrical engineering from Stanford University, Stanford, CA, and the Ph.D. degree in applied physics from California Institute of Technology, Pasadena.

He was the Founder of Telaris, Inc., Santa Monica, CA, where he is currently the Chief Technology Officer. Prior to Telaris, he founded Arroyo Optics, Inc., where he was the Vice President of R\&D. He has more than ten years experience in developing and commercializing a wide range of optical components and subsystems for the telecommunications industry. He is the holder of over 30 awarded or pending patents in the area of optoelectronics.

George Rakuljic received the M.S. and Ph.D. degrees in electrical engineering from California Institute of Technology, Pasadena, in 1983 and 1987, respectively.

He was the Founder of Telaris, Inc., Santa Monica, CA, where he is currently the Chief Executive Officer. Prior to Telaris, He founded Arroyo Optics, Inc., where he was the Chief Technology Officer. He was the Vice President of R\&D at Accuwave Corporation and a Member of the Research Staff at the Northrop Research and Technology Center. He has numerous publications and patents in the fields of optics, materials science, and communications, and has been responsible for the development of several optoelectronic products for the telecommunications industry.

Amnon Yariv (S'56-M'59-F'70-LF'95) received the B.S., M.S., and Ph.D degrees in electrical engineering from the University of California, Berkeley, in 1954, 1956, and 1958, respectively.

In 1959, he joined Bell Telephone Laboratories, Murray Hill, NJ. In 1964, he joined California Institute of Technology (Caltech), Pasadena, as an Associate Professor of electrical engineering, where he became a Professor in 1966, the Thomas G. Myers Professor of electrical engineering and applied physics in 1980, and the Martin and Eileen Summerfield Professor of applied physics and Professor of electrical engineering in 1996. On the technical and scientific sides, he took part (with various coworkers) in the discovery of a number of early solid-state laser systems, in the original formulation of the theory of nonlinear quantum optics, in proposing and explaining mode-locked ultrashort-pulse lasers, GaAs optoelectronics, in proposing and demonstrating semiconductorbased integrated optics technology, in pioneering the field of phase conjugate optics, and in proposing and demonstrating the semiconductor distributed feedback laser. He has published widely in the laser and optics fields, and has written a number of basic texts in quantum electronics, optics, and quantum mechanics.

Dr. Yariv is a member of the American Academy of Arts and Sciences, the National Academy of Engineering, and the National Academy of Sciences. 